\title{
Differential equations with random Gamma distributed moments of non-instantaneous impulses and p-moment exponential stability
}

https://doi.org/10.1515/dema-2018-0016

Received March 29, 2018; accepted July 11, 2018

\begin{abstract}
Nonlinear differential equations with impulses occurring at random time and acting noninstantaneously on finite intervals are studied. We consider the case when the time where the impulses occur is Gamma distributed. Lyapunov functions are applied to obtain sufficient conditions for the p-moment exponential stability of the trivial solution of the given system.
\end{abstract}

Keywords: fractional differential equations, random moments of impulses, non-instantaneous impulses, p-moment exponential stability

MSC: 34A08, 34A37, 34D20, 34F05

\section{Introduction}

Impulsive effects exist in many evolution processes when the states change abruptly at certain moments of time. These equations arise naturally in models in medicine, biology, economics, mechanics and electronics. In the literature many authors consider impulsive differential equations with determined impulsive moments and there are two popular types of deterministic impulses:

- instantaneous impulses: the duration of the changes is relatively short compared to the overall duration of the whole process. The model is given by impulsive differential equations (see for example, [1-6], the monographs $[7,8]$ and the references cited therein);

- non-instantaneous impulses - an impulsive action, which starts at an arbitrary fixed point and remains active on a finite time interval. E. Hernandez and D. O'Regan [9] introduced a new class of abstract differential equations for which the impulses are not instantaneous and they investigated the existence of mild and classical solutions. As a motivation for the study of this type we consider the following simplified situation concerning the hemodynamical equilibrium of a person. In the case of a decompensation (for example, high or low levels of glucose) one can prescribe some intravenous drugs (insulin). Since the introduction of the drugs in the bloodstream and the consequent absorption for the body are gradual and continuous processes, we can interpret the situation as an impulsive action which starts abruptly and stays active on a finite time interval. Recently results concerning non-instantaneous impulses were obtained for differential

Ravi Agarwal: Department of Mathematics, Texas A\&M University-Kingsville, Kingsville, TX 78363, USA Distinguished University Professor of Mathematics, Florida Institute of Technology, Melbourne, FL 32901, USA, E-mail: Ravi.Agarwal@tamuk.edu

*Corresponding Author: Snezhana Hristova: Department of Applied Mathematics and Modeling, University of Plovdiv, Plovdiv, Bulgaria, E-mail: snehri@gmail.com

Donal O'Regan: School of Mathematics, Statistics and Applied Mathematics, National University of Ireland, Galway, Ireland, E-mail: donal.oregan@nuigalway.ie

Peter Kopanov: Department of Applied Mathematics and Modeling, University of Plovdiv, Plovdiv, Bulgaria, E-mail:

pkopanov@yahoo.com 
equations [10-12], delay integro-differential equations [13], abstract differential equations [9], and fractional differential equations [14-16].

In some real world phenomena the process changes at uncertain moments. Modeling such real world phenomena where the state of the process under investigation changes instantaneously at uncertain moments will lead to combining ideas in differential equations and probability theory. When there is uncertainty in the behavior of the state of the investigated process an appropriate model is usually a stochastic differential equation where one or more of the terms in the differential equation are stochastic processes, and this usually results with the solution being a stochastic process [17-20]. Differential equations with instantaneously acting impulses at random times were studied in [21, 22] but there are some inaccuracies in the mixing properties of deterministic variables and random variables, and inaccuracies in the convergence of a sequence of real numbers to a random variable.

In this paper we study nonlinear differential equations subject to impulses starting abruptly at some random points and their action continue on intervals with a given finite length. We study the case of Gamma distributed random variables between two consecutive moments of impulses and the interval where the impulses act is of a constant length. The p-moment exponential stability of the solution is studied using Lyapunov functions.

\section{Non-instantaneous impulses in differential equations}

Initially, we will give a brief overview of differential equations with deterministic non-instantaneous impulses.

Let $T_{0} \geq 0$ be a given point and the increasing sequence of positive points $\left\{T_{k}\right\}_{k=1}^{\infty}$ and the sequence of non-negative numbers $\left\{d_{i}\right\}_{i=1}^{\infty}$ be given, such that $\lim _{k \rightarrow \infty} T_{k}=\infty$. Denote $d_{0}=0$.

Consider the initial value problem (IVP) for the system of non-instantaneous impulsive differential equations (NIDE) with fixed points of impulses

$$
\begin{aligned}
& x^{\prime}=f(t, x(t)) \quad \text { for } t \in\left[T_{k}+d_{k}, T_{k+1}\right], k=0,1,2, \ldots, \\
& x(t)=I_{k}\left(t, x\left(T_{k}-0\right)\right) \quad \text { for } t \in\left(T_{k}, T_{k}+d_{k}\right], k=1,2, \ldots, \\
& x\left(T_{0}\right)=x_{0},
\end{aligned}
$$

where $x, x_{0} \in \mathbb{R}^{n}, f:[0, \infty) \times \mathbb{R}^{n} \rightarrow \mathbb{R}^{n}, I_{i}:\left[T_{i}, T_{i}+d_{i}\right] \times \mathbb{R}^{n} \rightarrow \mathbb{R}^{n},(i=1,2,3, \ldots)$.

The solution of NIDE (1) depends not only on the initial condition $\left(T_{0}, x_{0}\right)$ but on the moments of impulses $T_{k}, k=1,2, \ldots$ and we will denote it by $x\left(t ; T_{0}, x_{0},\left\{T_{k}\right\}\right)$. We will assume that

$$
x\left(T_{k} ; T_{0}, x_{0},\left\{T_{k}\right\}\right)=\lim _{t \rightarrow T_{k}-0} x\left(t ; T_{0}, x_{0},\left\{T_{k}\right\}\right)
$$

for any $k=1,2, \ldots$ and

$$
\lim _{t \rightarrow T_{k}+d_{k}-0} x\left(t ; T_{0}, x_{0},\left\{T_{k}\right\}\right)=\lim _{t \rightarrow T_{k}+d_{k}+0} x\left(t ; T_{0}, x_{0},\left\{T_{k}\right\}\right) \quad \text { for any } k=1,2, \ldots
$$

We will assume the following conditions are satisfied

H1. $f(t, 0)=0$ and $I_{k}(t, 0)=0$ for $t \geq 0, k=1,2, \ldots$

H2. For any initial value $\left(T_{0}, x_{0}\right)$ the ODE $x^{\prime}=f(t, x)$ with $x\left(T_{0}\right)=x_{0}$ has a unique solution $x(t)=$ $x\left(t ; T_{0}, x_{0}\right)$ defined for $t \geq T_{0}$.

Example 1. (differential equations with fixed points of non-instantaneous impulses). Consider the following IVP for the scalar NIDE

$$
\begin{aligned}
& x^{\prime}=a_{k} x \text { for } t \in\left[T_{k}+d_{k}, T_{k+1}\right], k=0,1,2, \ldots, \\
& x(t)=I_{k}\left(t, x\left(T_{k}-0\right)\right) \text { for } t \in\left(T_{k}, T_{k}+d_{k}\right], k=1,2, \ldots, \\
& x\left(T_{0}\right)=x_{0},
\end{aligned}
$$

where $x, x_{0} \in \mathbb{R}, a_{k}, k=0,1,2, \ldots$, are constants. 
The solution $x(t)=x\left(t ; T_{0}, x_{0},\left\{T_{k}\right\}\right)$ of $(2)$ is

$$
x(t)=\left\{\begin{array}{l}
x_{0} e^{a_{0}\left(t-T_{0}\right)} \text { for } t \in\left[T_{0}, T_{1}\right], \\
I_{k}\left(t, x\left(T_{k}-0\right)\right) \text { for } t \in\left(T_{k}, T_{k}+d_{k}\right], k=1,2, \ldots, \\
I_{k}\left(T_{k}+d_{k}, x\left(T_{k}-0\right)\right) e^{a\left(t-T_{k}-d_{k}\right)} \text { for } t \in\left[T_{k}+d_{k}, T_{k+1}\right], k=1,2,3, \ldots
\end{array}\right.
$$

If $I_{k}(t, x)=b_{k}(t) x, b_{k}:\left[T_{k}, T_{k}+d_{k}\right] \rightarrow \mathbb{R} /\{1\}, k=1,2,3, \ldots$, then the solution of NIDE (2) is given by (note the product is 1 if $k=1$ )

$$
x(t)=\left\{\begin{array}{l}
x_{0} e^{a_{0}\left(t-T_{0}\right)} \text { for } t \in\left[T_{0}, T_{1}\right], \\
x_{0} e^{\sum_{i=0}^{k-1} a_{i}\left(T_{i+1}-T_{i}-d_{i}\right)}\left(\prod_{i=1}^{k-1} b_{i}\left(T_{i}+d_{i}\right)\right) b_{k}(t) \text { for } t \in\left(T_{k}, T_{k}+d_{k}\right], \\
x_{0} e^{\sum_{i=0}^{k-1} a_{i}\left(T_{i+1}-T_{i}-d_{i}\right)}\left(\prod_{i=1}^{k} b_{i}\left(T_{i}+d_{i}\right)\right) e^{a_{k}\left(t-T_{k}-d_{k}\right)} \text { for } t \in\left[T_{k}+d_{k}, T_{k+1}\right] .
\end{array}\right.
$$

If $b_{k}(t)=e^{a_{k} t}, k=1,2,3, \ldots$ the solution of $\operatorname{NIDE}(2)$ is given by

$$
x(t)=\left\{\begin{array}{l}
x_{0} e^{a_{0}\left(t-T_{0}\right)} \text { for } t \in\left[T_{0}, T_{1}\right], \\
x_{0} e^{a_{k} t-a_{0} T_{0}+\sum_{i=0}^{k-1} a_{i} T_{i+1}} \text { for } t \in\left(T_{k}, T_{k}+d_{k}\right], k=1,2, \ldots, \\
x_{0} e^{a_{k} t-a_{0} T_{0}+\sum_{i=0}^{k-1} a_{i} T_{i+1}} \text { for } t \in\left[T_{k}+d_{k}, T_{k+1}\right], k=1,2, \ldots
\end{array}\right.
$$

If $a_{k}=a, k=0,1, \ldots$ and $I_{k}(t, x)=b x$ with $b \neq 1, k=1,2,3, \ldots$ is a constant, then the solution of $\operatorname{NIDE}(2)$ is

$$
x(t)=\left\{\begin{array}{l}
x_{0} e^{a\left(t-T_{0}\right)} \text { for } t \in\left[T_{0}, T_{1}\right], \\
x_{0} b^{k} e^{a \sum_{i=0}^{k-1}\left(T_{i+1}-S_{k}\right)} \text { for } t \in\left(T_{k}, T_{k}+d_{k}\right], k=1,2, \ldots, \\
x_{0} b^{k} e^{a \sum_{i=0}^{k-1}\left(T_{i+1}-T_{k}-d_{k}\right)} e^{a\left(t-T_{k}-d_{k}\right)} \text { for } t \in\left[T_{k}+d_{k}, T_{k+1}\right], k=1,2, \ldots
\end{array}\right.
$$

If $a_{k}=0, k=0,1, \ldots$ and $I_{k}(t, x)=b_{k}(t) x, b_{k}:\left[t_{k}, s_{k}\right] \rightarrow \mathbb{R} /\{1\}, k=1,2,3, \ldots$, then the solution of NIDE (2) is

$$
x(t)=\left\{\begin{array}{l}
x_{0} \text { for } t \in\left[T_{0}, T_{1}\right], \\
x_{0}\left(\prod_{i=1}^{k-1} b_{i}\left(s_{i}\right)\right) b_{k}(t) \text { for } t \in\left(T_{k}, s_{k}\right], k=1,2, \ldots \\
x_{0}\left(\prod_{i=1}^{k} b_{i}\left(s_{i}\right)\right) \text { for } t \in\left[s_{k}, T_{k+1}\right], k=1,2,3, \ldots
\end{array}\right.
$$

In Section 6 we will need the following result for the initial value problem for a scalar linear differential inequality with non-instantaneous fixed moments of impulses:

$$
\begin{aligned}
& u^{\prime} \leq-m_{k} u \text { for } T_{k}+d_{k} \leq t \leq T_{k+1}, k=0,1,2, \ldots, \\
& u(t) \leq b_{k} u\left(T_{k}-0\right) \text { for } T_{k}<t \leq T_{k}+d_{k}, k=1,2, \ldots, \\
& u\left(T_{0}\right) \leq 0 .
\end{aligned}
$$

Proposition 1. Let $m_{k}>0(k=0,1,2 \ldots)$ and $b_{k}>0(k=1,2, \ldots)$ be real constants. Then $m(t) \leq 0$ for $t \geq T_{0}$.

Proof: Let $t \in\left[T_{0}, T_{1}\right]$. Then the function $u(t)$ is continuous on $\left[T_{0}, T_{1}\right]$ and $u(t) \leq u\left(T_{0}\right) e^{-m_{0}\left(t-T_{0}\right)} \leq 0$.

Let $t \in\left(T_{1}, T_{1}+d_{1}\right]$. Then the function $u(t) \leq b_{1} u\left(T_{1}-0\right) \leq 0$.

Let $t \in\left[T_{1}+d_{1}, T_{2}\right]$. Then the function $u(t)$ is continuous on $\left[T_{1}+d_{1}, T_{2}\right]$ and $u(t) \leq u\left(T_{1}+\right.$ $\left.d_{1}\right) e^{-m_{1}\left(t-T_{1}-d_{1}\right)} \leq 0$.

Continue this process. 


\section{Random non-instantaneous impulses in differential equations}

Let the probability space $(\Omega, \mathcal{F}, P)$ be given. Let $\left\{\tau_{k}\right\}_{k=1}^{\infty}$ be a sequence of random variables defined on the sample space $\Omega$.

Assume $\sum_{k=1}^{\infty} \tau_{k}=\infty$ with probability 1.

Remark 1. The random variables $\tau_{k}$ will define the time between two consecutive impulsive moments of the impulsive differential equation with random impulses.

We will assume the following condition is satisfied:

H3. The random variables $\left\{\tau_{k}\right\}_{k=1}^{\infty}$ are independent Gamma distributed random variables with the same rate parameter $\lambda$ and shape parameter $\alpha_{k}$ respectively, i.e., $\tau_{k} \in \Gamma\left(\alpha_{k}, \lambda\right)$ with $\lambda>0, \alpha_{k}>0$.

We recall some properties of the Gamma distribution:

(i) If $X \in \operatorname{Exp}(\lambda)$, then $X \in \Gamma(1, \lambda)$;

(ii) If $X \in \Gamma\left(\alpha_{1}, c\right)$ and $Y \in \Gamma\left(\alpha_{2}, c\right)$ are independent, then $X+Y \in \Gamma\left(\alpha_{1}+\alpha_{2}, c\right)$;

(iii) The cumulative distribution function (CDF) of $X \in \Gamma(\alpha, c)$ is

$$
F(x ; \alpha, c)=P(X \leq x)=\frac{\gamma(\alpha, c x)}{\Gamma(\alpha)} \text { for } x>0
$$

and the density function (DF) is

$$
f(x ; \alpha, c)=\frac{c^{\alpha} x^{\alpha-1} e^{-c x}}{\Gamma(\alpha)} \text { for } x>0,
$$

where $\gamma(A, t)=\int_{0}^{t} x^{A-1} e^{-x} d x, A, t>0$, is the lower incomplete Gamma function. Note that there is an equivalent presentation of the lower incomplete Gamma function

$$
\gamma(A, t)=t^{A} e^{-t} \Gamma(A) \sum_{j=0}^{\infty} \frac{t^{j}}{\Gamma(A+j+1)} .
$$

Using the properties of the Gamma distribution we obtain the following result:

Proposition 2. Let condition (H3) be satisfied and the sequence of random variables $\left\{\Xi_{k}\right\}_{k=1}^{\infty}$ be such that $\Xi_{n}=\sum_{i=1}^{n} \tau_{i}, n=1,2, \ldots$ Then $\Xi_{n} \in \Gamma\left(\sum_{i=1}^{n} \alpha_{i}, \lambda\right)$, i.e., the cdf of $\Xi_{n}$ is

$$
F\left(t ; \sum_{i=1}^{n} \alpha_{i}, \lambda\right)=P\left(\Xi_{n}<t\right)= \begin{cases}\frac{\gamma\left(\sum_{i=1}^{n} \alpha_{i}, t \lambda\right)}{\Gamma\left(\sum_{i=1}^{n} \alpha_{i}\right)} & \text { for } t>0, \\ 0 & \text { for } t \leq 0 .\end{cases}
$$

Corollary 1. If condition (H3) is satisfied for $\alpha_{k}=1, k=1,2 \ldots$, i.e., $\tau_{k} \in \operatorname{Exp}(\lambda)$, then the cdf of $\Xi_{n}$ is

$$
F(t ; \lambda)=P\left(\Xi_{n}<t\right)= \begin{cases}1-e^{-\lambda t} \sum_{j=0}^{n-1} \frac{(\lambda t)^{j}}{j !} & \text { for } t>0, \\ 0 & \text { for } t \leq 0 .\end{cases}
$$

Let the sequence of positive numbers $\left\{d_{k}\right\}_{k=1}^{\infty}$ be given. Denote $d_{0}=0$.

Consider the following conditions:

H4. The positive numbers $\left\{d_{k}\right\}_{k=1}^{\infty}$ are such that $\lim _{n \rightarrow \infty} \sum_{k=1}^{n} d_{k}=\infty$.

H5. The positive numbers $\left\{d_{k}\right\}_{k=1}^{\infty}$ are such that $\lim _{n \rightarrow \infty} \sum_{k=1}^{n} d_{k}=B<\infty$.

Remark 2. Note that if there exist constants $c>0, s \leq 1$ and for any $k=1,2, \ldots$ at least one of inequalities $d_{k} \geq \frac{c}{s^{k}}$ or $d_{k} \geq \frac{c}{k^{s}}$ holds, then condition (H4) is satisfied.

If $c=d>0, s=1$ and $d_{k}=d>0$, or $c=1, s=1, d_{k}=\frac{1}{k}, k=1,2, \ldots$, then condition (H4) is satisfied.

If there exist constants $c>0, s>1$ and for any $k=1,2, \ldots$ at least one of inequalities $d_{k} \leq \frac{c}{s^{k}}$ or $d_{k} \leq \frac{c}{k^{s}}$ holds, then condition (H5) is satisfied.

If $c=1, s=2$ and $d_{k}=\frac{1}{2^{k}}, k=1,2, \ldots$, then condition (H5) is satisfied. 
Define the sequence of random variables $\left\{\xi_{k}\right\}_{k=0}^{\infty}$ by

$$
\xi_{k}=T_{0}+\sum_{i=1}^{k} \tau_{i}+\sum_{i=1}^{k-1} d_{i}, \quad k=0,1,2, \ldots
$$

where $T_{0} \geq 0$ is a fixed point.

The random variable $\tau_{k}$ measures the waiting time of the $k$-th impulse after the $(k-1)$-st impulse occurs and stops its action, $d_{k}$ is the length of the acting time interval of the $k$-th impulse and the random variable $\xi_{k}$ denotes the length of time until $k$ impulses occur for $t \geq T_{0}$.

We note that $\left\{\xi_{k}\right\}_{k=0}^{\infty}$ is a sequence of random variables defined by the recurrence formula $\xi_{k}=\xi_{k-1}+\tau_{k}+$ $d_{k-1}, k=1,2, \ldots$ with $d_{0}=0$.

Remark 3. The random variable $\xi_{n}$ will be called the waiting time and it gives the arrival time of $n$-th impulses in the impulsive differential equation with random impulses.

Also, $\Xi_{k}=\sum_{i=1}^{n} \tau_{i}=\xi_{k}-T_{0}-\sum_{i=1}^{k-1} d_{i}, k=1,2, \ldots$

Let the points $t_{k}$ be arbitrary values of the corresponding random variables $\tau_{k}, k=1,2, \ldots$ Define two increasing sequences of points

$$
T_{k}=T_{0}+\sum_{i=1}^{k} t_{i}+\sum_{i=1}^{k-1} d_{i}, \quad k=1,2,3 \ldots
$$

and

$$
s_{k}=T_{k}+d_{k}, \quad k=1,2,3 \ldots
$$

Note that $T_{k}$ and $s_{k}$ are values of the corresponding random variables $\xi_{k}$ and $\xi_{k}+d_{k}$ and $s_{k-1} \leq T_{k} \leq s_{k}$. Then the initial value problem for the system of NIDE with fixed points of impulses (1) reduces to

$$
\begin{aligned}
& x^{\prime}=f(t, x(t)) \text { for } t \in\left(T_{k}+d_{k}, T_{k+1}\right], k=0,1,2, \ldots, \\
& x(t)=I_{k}\left(t, x\left(T_{k}-0\right)\right) \text { for } t \in\left(T_{k}, T_{k}+d_{k}\right], k=1,2, \ldots, \\
& x\left(T_{0}\right)=x_{0},
\end{aligned}
$$

where $x, x_{0} \in \mathbb{R}^{n}, f:[0, \infty) \times \mathbb{R}^{n} \rightarrow \mathbb{R}^{n}, I_{i}:\left[t_{i}, s_{i}\right] \times \mathbb{R}^{n} \rightarrow \mathbb{R}^{n},(i=1,2,3, \ldots)$.

The set of all solutions $x\left(t ; T_{0}, x_{0},\left\{T_{k}\right\}\right)$ of NIDE (9) for any values $t_{k}$ of the random variables $\tau_{k}$, $k=1,2, \ldots$ generates a specific stochastic process with state space $\mathbb{R}^{n}$. We denote it by $x\left(t ; T_{0}, x_{0},\left\{\tau_{k}\right)\right\}$ and we will say that it is a solution of the following initial value problem for non-instantaneous impulsive differential equations with random moments of impulses (RNIDE)

$$
\begin{aligned}
& x^{\prime}(t)=f(t, x(t)) \text { for } t \geq T_{0}, \xi_{k}+d_{k}<t<\xi_{k+1}, k=0,1, \ldots, \\
& x(t)=I_{k}\left(t, x\left(\xi_{k}\right)\right), \text { for } \xi_{k}<t<\xi_{k}+d_{k}, k=1,2, \ldots, \\
& x\left(T_{0}\right)=x_{0},
\end{aligned}
$$

where $x_{0} \in \mathbb{R}^{n}$.

Definition 1. Let $t_{k}$ be a value of the random variable $\tau_{k}, k=1,2,3, \ldots$ and $T_{k}=T_{0}+\sum_{i=1}^{k} t_{i}, k=1,2, \ldots$ Then the solution $x\left(t ; T_{0}, x_{0},\left\{T_{k}\right\}\right)$ of the IVP for the IDE with fixed points of impulses (1) is called a sample path solution of the IVP for the RIDE (10).

Any sample path solution

$$
x\left(t ; T_{0}, x_{0},\left\{T_{k}\right\}\right) \in C^{1}\left(\left(T_{k}, T_{k+1}\right], \mathbb{R}^{n}\right),
$$

for $k=0,1,2, \ldots$

Definition 2. A stochastic process $x\left(t ; T_{0}, x_{0},\left\{\tau_{k}\right)\right\}$ with an uncountable state space $\mathbb{R}^{n}$ is said to be a solution of the IVP for the system of RIDE (10) if for any values $t_{k}$ of the random variable $\tau_{k}, k=1,2,3, \ldots$ and $T_{k}=T_{0}+\sum_{i=1}^{k} t_{i}, k=1,2, \ldots$ the corresponding function $x\left(t ; T_{0}, x_{0},\left\{T_{k}\right\}\right)$ is a sample path solution of the IVP for RIDE (10). 


\section{Preliminary results for Gamma distributed moments of impulses}

We will use the following probability result:

Lemma 4.1. Let the continuous random variables $\xi$, $\eta$ be such that $\xi \geq \eta$. Then for any $t \geq 0$ the equality

$$
P(\eta<t<\xi)=F_{\eta}(t)-F_{\xi}(t)
$$

holds.

Proof: Using continuity of the random variables $\eta$ and $\xi$ we obtain

$$
P(\eta<t<\xi)=P(\eta<t \cap \xi>t)=P(\eta<t \backslash(\eta<t \cap \xi<t))=P(\eta<t \backslash \xi<t)=P(\eta<t)-P(\xi<t) .
$$

For any $t \geq T_{0}$ consider the events

$$
S_{0}(t)=\left\{\omega \in \Omega: t-T_{0}<\tau_{1}(\omega)\right\}
$$

and

$$
S_{k}(t)=\left\{\omega \in \Omega: \xi_{k}(\omega)+d_{k}<t<\xi_{k+1}(\omega)\right\}, \quad k=1,2, \ldots,
$$

where the random variables $\xi_{k}, k=1,2, \ldots$ are defined by (8).

In the case of Gamma distributed random variables $\tau_{k}, k=1,2, \ldots$ we obtain the following results:

Proposition 3. For any $t \geq T_{0}$ the equality $P\left(S_{0}(t)\right)=1-\frac{\gamma\left(\alpha_{1}, \lambda\left(t-T_{0}\right)\right)}{\Gamma\left(\alpha_{1}\right)}$ holds.

Lemma 4.2. Let conditions (H3),(H4) be satisfied and $t \geq T_{0}$ be a given fixed number. Then the probability that there will be exactly $k$ impulses until time $t$ is

$$
P\left(S_{k}(t)\right)=\left\{\begin{array}{l}
0 \quad \text { for } k>C_{t}, \\
\frac{\gamma\left(\sum_{i=1}^{k} \alpha_{i},\left(t-T_{0}-\sum_{i=1}^{k} d_{i}\right) \lambda\right)}{\Gamma\left(\sum_{i=1}^{k} \alpha_{i}\right)}-\frac{\gamma\left(\sum_{i=1}^{k+1} \alpha_{i},\left(t-T_{0}-\sum_{i=1}^{k} d_{i}\right) \lambda\right)}{\Gamma\left(\sum_{i=1}^{k+1} \alpha_{i}\right)} \text { for } k \leq C_{t},
\end{array}\right.
$$

where $C_{t}=\min \left\{c \in \mathbb{Z}: t \leq T_{0}+\sum_{i=1}^{c} d_{i}\right\}$.

Proof: Let $t>T_{0}$ be fixed. According to condition (H4) there exists a natural number $C_{t}$, such that $T_{0}+\sum_{i=1}^{C_{t}-1} d_{i}<t \leq T_{0}+\sum_{i=1}^{C_{t}} d_{i}$. Consider the sequence of random variables $\Xi_{k}, k \geq 1$, defined as above. For any natural number $k$, by Lemma 4.1, we obtain

$$
\begin{aligned}
P\left(S_{k}(t)\right) & =P\left(T_{0}+\sum_{i=1}^{k} \tau_{i}+\sum_{i=1}^{k} d_{i}<t<T_{0}+\sum_{i=1}^{k+1} \tau_{i}+\sum_{i=1}^{k} d_{i}\right) \\
& =P\left(\Xi_{k}<t-T_{0}-\sum_{i=1}^{k} d_{i}\right)-P\left(\Xi_{k+1}<t-T_{0}-\sum_{i=1}^{k} d_{i}\right) .
\end{aligned}
$$

If $k>C_{t}$, then $t<T_{0}+\sum_{i=1}^{k} d_{i}$ and $P\left(S_{k}(t)\right)=0$.

Now if $k \leq C_{t}$, then $t-T_{0}-\sum_{i=1}^{k} d_{i} \geq 0$ and from (12) and Proposition 2 we get

$$
\begin{aligned}
P\left(S_{k}(t)\right) & =F_{\Xi_{k}}\left(t-T_{0}-\sum_{i=1}^{k} d_{i} ; \sum_{i=1}^{k} \alpha_{i}, \lambda\right)-F_{\Xi_{k+1}}\left(t-T_{0}-\sum_{i=1}^{k} d_{i} ; \sum_{i=1}^{k+1} \alpha_{i}, \lambda\right) \\
& =\frac{\gamma\left(\sum_{i=1}^{k} \alpha_{i},\left(t-T_{0}-\sum_{i=1}^{k} d_{i}\right) \lambda\right)}{\Gamma\left(\sum_{i=1}^{k} \alpha_{i}\right)}-\frac{\gamma\left(\sum_{i=1}^{k+1} \alpha_{i},\left(t-T_{0}-\sum_{i=1}^{k} d_{i}\right) \lambda\right)}{\Gamma\left(\sum_{i=1}^{k+1} \alpha_{i}\right)} .
\end{aligned}
$$


Corollary 2. Let condition (H3), (H4) be satisfied with $\alpha_{i}=\alpha, i=1,2, \ldots$ and $t \geq T_{0}$ be a given fixed number. Then

$$
P\left(S_{k}(t)\right)=\left\{\begin{array}{l}
0 \text { for } k>C_{t}, \\
\int_{0}^{\left(t-T_{0}-\sum_{i=1}^{k} d_{i}\right) \lambda}\left(\frac{x^{k \alpha-1}}{\Gamma(k \alpha)}-\frac{\chi^{(k+1) \alpha-1}}{\Gamma((k+1) \alpha)}\right) e^{-x} d x \quad \text { for } k \leq C_{t},
\end{array}\right.
$$

where $C_{t}=\min \left\{c \in \mathbb{Z}: t \leq T_{0}+\sum_{i=1}^{c} d_{i}\right\}$.

Corollary 3. If the condition (H3) is satisfied with $\alpha_{k}=1$, i.e., $\tau_{k} \in \operatorname{Exp}(\lambda)$, and $d_{k}=d>0, k=1,2, \ldots$, then

$$
P\left(S_{k}(t)\right)=\left\{\begin{array}{l}
0 \text { for } k>C_{t}, \\
e^{-\lambda\left(t-T_{0}\right)} \frac{\left(\lambda e^{\lambda d}\left(t-T_{0}-k d\right)\right)^{k}}{k !} \leq e^{-\lambda\left(t-T_{0}\right)} \frac{\left(\lambda e^{\lambda d}\left(t-T_{0}\right)\right)^{k}}{k !} \text { for } k \leq C_{t} .
\end{array}\right.
$$

The proof of Corollary 3 follows from Lemma 4.2 and equality $\gamma(k, t)-\frac{\gamma(k+1, t)}{k}=\frac{t^{k} e^{-t}}{k}$.

Lemma 4.3. (Upper bound of $S_{k}(t)$ ) Let conditions (H3),(H4) be satisfied. Then for any $k \leq C_{t}$ there exists a polynomial $R_{k}(x)$ (as described in the proof) with positive coefficients defined for $x \geq 0$, such that

$$
P\left(S_{k}(t)\right) \leq e^{-\left(t-T_{0}-\sum_{i=1}^{k} d_{i}\right) \lambda} R_{k}\left(\left(t-T_{0}-\sum_{i=1}^{k} d_{i}\right) \lambda\right) \text { for } t>T_{0}+\sum_{i=1}^{k} d_{i} .
$$

Proof: Let $k \leq C_{t}$ be a natural fixed number and $t>T_{0}+\sum_{i=1}^{k} d_{i}$. Denote $A_{k}=\sum_{i=1}^{k} d_{i}$ and $z=\left(t-T_{0}-\sum_{i=1}^{k} d_{i}\right) \lambda$. From Lemma 4.2 and the presentation (7) of the lower incomplete Gamma function we obtain

$$
\begin{aligned}
P\left(S_{k}(t)\right) & =z^{A_{k}} e^{-z} \sum_{j=0}^{\infty} \frac{z^{j}}{\Gamma\left(A_{k}+j+1\right)}-z^{A_{k+1}} e^{-z} \sum_{j=0}^{\infty} \frac{z^{j}}{\Gamma\left(A_{k+1}+j+1\right)} \\
& =e^{-z}\left[\sum_{j=0}^{\infty}\left(\frac{z^{A_{k}+j}}{\Gamma\left(A_{k}+j+1\right)}-\frac{z^{A_{k+1}+j}}{\Gamma\left(A_{k+1}+j+1\right)}\right)\right] \\
& =e^{-z} \sum_{j=0}^{N-1} \frac{z^{A_{k}+j}}{\Gamma\left(A_{k}+j+1\right)}+e^{-z}\left[\sum_{j=0}^{\infty}\left(\frac{z^{A_{k}+N+j}}{\Gamma\left(A_{k}+N+j+1\right)}-\frac{z^{A_{k+1}+j}}{\Gamma\left(A_{k+1}+j+1\right)}\right)\right] \\
& =e^{-z} \sum_{j=0}^{N-1} \frac{z^{A_{k}+j}}{\Gamma\left(A_{k}+j+1\right)}+e^{-z} \sum_{j=0}^{\infty} \frac{z^{A_{k}+N+j}}{\Gamma\left(A_{k}+N+j+1\right)}-\frac{\gamma\left(A_{k+1}, z\right)}{\Gamma\left(A_{k+1}\right)},
\end{aligned}
$$

where $N$ is an arbitrary natural number.

For any natural number $N$ we define two functions $f_{N}, g_{N}:[0, \infty) \rightarrow[0,1]$ by the equalities

$$
f_{N}(z)=e^{-z} \sum_{j=0}^{N-1} \frac{z^{A_{k}+j}}{\Gamma\left(A_{k}+j+1\right)}
$$

and

$$
g_{N}(z)=e^{-z} \sum_{j=0}^{\infty} \frac{z^{A_{k}+N+j}}{\Gamma\left(A_{k}+N+j+1\right)}-\frac{\gamma\left(A_{k+1}, z\right)}{\Gamma\left(A_{k+1}\right)} .
$$

Then from (14) we get

$$
P\left(S_{k}(t)\right)=f_{N}(z)+g_{N}(z)
$$

where $N$ is an arbitrary natural number. 
For any fixed $z \geq 0$ the sequence $\left\{f_{N}(z)\right\}_{N=1}^{\infty}$ is strictly increasing and for any natural $N$ we have the inequality $\sup _{z \geq 0} f_{N}(z) \leq 1$ and the function $f_{N}(z)$ is continuous in $[0, \infty)$. Therefore, the sequence $\left\{f_{N}(z)\right\}_{N=1}^{\infty}$ is convergent and $\lim _{N \rightarrow \infty} f_{N}(z)=F(z)$, where $F(z)=e^{-z} \sum_{j=0}^{\infty} \frac{z^{A_{k}+j}}{\Gamma\left(A_{k}+j+1\right)}$. Note that the function $F(z)$ is continuous on $[0, \infty)$ (see [23]).

Denote $A_{N}=\sup _{z \geq 0}\left|F(z)-f_{N}(z)\right|$. The sequence $\left\{A_{N}\right\}_{N=1}^{\infty}$ in decreasing and therefore it is convergent. From the definition of the function $F(z)$ the equality $\lim _{N \rightarrow \infty} A_{N}=0$ follows, i.e., $F(z)$ is uniformly continuous on $[0, \infty)$.

Take the limit as $N \rightarrow \infty$ in (15) and we obtain

$$
P\left(S_{k}(t)\right)=e^{-z} \sum_{j=0}^{\infty} \frac{z^{A_{k}+j}}{\Gamma\left(A_{k}+j+1\right)}+\lim _{N \rightarrow \infty} g_{N}(z) \text { for } z \geq 0 .
$$

From (14) and (16) we get

$$
\lim _{N \rightarrow \infty} g_{N}(z)=-z^{A_{k+1}} e^{-z} \sum_{j=0}^{\infty} \frac{z^{j}}{\Gamma\left(A_{k+1}+j+1\right)}<0 \text { for } z>0 .
$$

Then, there exists a natural number $N_{0}>0$, such that for any $N \geq N_{0}$ the inequality $g_{N}(z)<0$ holds. Then from (15) and $z^{a} \leq z^{[a]}+z^{[a]+1}, z \geq 0$, the inequality

$$
P\left(S_{k}(t)\right)=f_{N}(z)+g_{N}(z)<f_{N}(z)=e^{-z} \sum_{j=0}^{N-1} \frac{z^{A_{k}+j}}{\Gamma\left(A_{k}+j+1\right)} \leq e^{-z} \sum_{j=0}^{N-1} \frac{z^{\left[A_{k}\right]+j}+z^{\left[A_{k}\right]+j+1}}{\Gamma\left(A_{k}+j+1\right)} \text { for } N \geq N_{0}
$$

holds, where $[a]$ in the integer part of the real number $a>0$.

If $N=N_{0}$, then from the inequality (17) we obtain

$$
P\left(S_{k}(t)\right) \leq e^{-z} \sum_{j=0}^{N_{0}-1} \frac{z^{\left[A_{k}\right]+j}+z^{\left[A_{k}\right]+j+1}}{\Gamma\left(A_{k}+j+1\right)} .
$$

Since the coefficients in the power series are positive numbers, there exists a polynomial $R_{k}(z)$ with a degree not more than $N_{0}+\left[A_{k}\right]+1$, such that

$$
\sum_{j=0}^{N_{0}-1} \frac{z^{\left[A_{k}\right]+j}+z^{\left[A_{k}\right]+j+1}}{\Gamma\left(A_{k}+j+1\right)} \leq R_{k}(z)
$$

and

$$
P\left(S_{k}(t)\right) \leq e^{-z} R_{k}(z)
$$

Corollary 4. Let conditions (H3),(H4) be satisfied. Then for any $k=1,2, \ldots$ and any positive number $c>0$ there exist numbers $M_{k}>0$ and $K_{k}>0$ depending on both $k$ and $c$, such that

$$
P\left(S_{k}(t)\right) \leq e^{-\lambda\left(t-T_{0}\right)} K_{k}\left(t-T_{0}\right)^{M_{k}} \quad \text { for } t \geq T_{0}+c .
$$

Proof: Let $k$ be a natural number and $c>0$ be a number. From Lemma 4.3 there exists a polynomial $R_{k}(x)$ (as described in the proof of Lemma 4.3) with positive coefficients defined for $x \geq 0$, such that (13) is satisfied. Consider the polynomial $R_{k}\left(\left(t-T_{0}-\sum_{i=1}^{k} d_{i}\right) \lambda\right)$ which could be presented as a polynomial depending on $t-T_{0}$, i.e., there exists a polynomial $P_{k}(x)$ of degree not more than the degree of $R_{k}(x)$, such that $P_{k}\left(t-T_{0}\right)=$ $R_{k}\left(\left(t-T_{0}-\sum_{i=1}^{k} d_{i}\right) \lambda\right)$. Also for $t>T_{0}+c$ there exists numbers $M_{k}=M(k, c)>0$ and $K_{k}=K(k, c)>0$, such that $P_{k}\left(t-T_{0}\right) \leq K_{k}\left(t-T_{0}\right)^{M_{k}}$. Then

$$
P\left(S_{k}(t)\right) \leq e^{-\lambda\left(t-T_{0}\right)} e^{\lambda \sum_{i=1}^{k} d_{i}} K_{k}\left(t-T_{0}\right)^{M_{k}}
$$

for $t>T_{0}+c$. 
Lemma 4.4. Let conditions (H3),(H5) be satisfied and $t \geq T_{0}$ be a given fixed number. Then the probability that there will be exactly $k$ impulses until time $t$ is:

- for $t \geq B$ :

$$
P\left(S_{k}(t)\right)=\frac{\gamma\left(\sum_{i=1}^{k} \alpha_{i},\left(t-T_{0}-\sum_{i=1}^{k} d_{i}\right) \lambda\right)}{\Gamma\left(\sum_{i=1}^{k} \alpha_{i}\right)}-\frac{\gamma\left(\sum_{i=1}^{k+1} \alpha_{i},\left(t-T_{0}-\sum_{i=1}^{k} d_{i}\right) \lambda\right)}{\Gamma\left(\sum_{i=1}^{k+1} \alpha_{i}\right)} ;
$$

- for $t<B$ :

$$
P\left(S_{k}(t)\right)=\left\{\begin{array}{l}
0 \quad \text { for } k>C_{t}, \\
\frac{\gamma\left(\sum_{i=1}^{k} \alpha_{i},\left(t-T_{0}-\sum_{i=1}^{k} d_{i}\right) \lambda\right)}{\Gamma\left(\sum_{i=1}^{k} \alpha_{i}\right)}-\frac{\gamma\left(\sum_{i=1}^{k+1} \alpha_{i},\left(t-T_{0}-\sum_{i=1}^{k} d_{i}\right) \lambda\right)}{\Gamma\left(\sum_{i=1}^{k+1} \alpha_{i}\right)} \text { for } k \leq C_{t},
\end{array}\right.
$$

where $C_{t}=\min \left\{c \in \mathbb{Z}: t \leq T_{0}+\sum_{i=1}^{c} d_{i}\right\}$.

The proof of Lemma 4.4 is similar to the one of Lemma 4.2, so we omit it.

Remark 4. The claim of Lemma 4.3 and Corollary 4 are true if condition (H4) is replaced by (H5). In the proof we use Lemma 4.4 instead of Lemma 4.2.

For any $t \geq T_{0}$ consider the events

$$
W_{k}(t)=\left\{\omega \in \Omega: \xi_{k}(\omega)<t<\xi_{k}(\omega)+d_{k}\right\}, k=1,2, \ldots,
$$

where the random variables $\xi_{k}, k=1,2, \ldots$ are defined as above.

In the case of Gamma distributed random variables $\tau_{k}, k=1,2, \ldots$ we obtain the following result:

Lemma 4.5. Let conditions (H3), (H4) hold and $t \geq T_{0}$ be a given fixed number. Then the probability the time $t$ is immediately after the $k$-th random impulse but not further away than $d_{k}$ from it is given by

$$
P\left(W_{k}(t)\right)= \begin{cases}0 \quad \text { for } k>C_{t}, & \\ \frac{\gamma\left(\sum_{i=1}^{k} \alpha_{i}, \lambda\left(t-T_{0}-\sum_{i=1}^{k-1} d_{i}\right)\right)}{\Gamma\left(\sum_{i=1}^{k} \alpha_{i}\right)}-\frac{\gamma\left(\sum_{i=1}^{k} \alpha_{i}, \lambda\left(t-T_{0}-\sum_{i=1}^{k} d_{i}\right)\right)}{\Gamma\left(\sum_{i=1}^{k} \alpha_{i}\right)} & \text { for } k \leq C_{t},\end{cases}
$$

where $C_{t}=\min \left\{c \in \mathbb{Z}: t \leq T_{0}+\sum_{i=1}^{c} d_{i}\right\}$.

Proof: Let $t \geq T_{0}$ be fixed. According to condition (H4) there exists a natural number $C_{t}$, such that $T_{0}+\sum_{i=1}^{C_{t}-1} d_{i}<t \leq T_{0}+\sum_{i=1}^{C_{t}} d_{i}$. Consider the sequence of random variables $\Xi_{k}, k \geq 1$, defined as above. For any natural $k$, by Lemma 4.1, we obtain

$$
\begin{aligned}
P\left(W_{k}(t)\right) & =P\left(\xi_{k}<t<\xi_{k}+d_{k}\right)=P\left(t-T_{0}-\sum_{i=1}^{k} d_{i}<\sum_{i=1}^{k} \tau_{i}<t-T_{0}-\sum_{i=1}^{k-1} d_{i}\right) \\
& =F_{\Xi_{k}}\left(t-T_{0}-\sum_{i=1}^{k-1} d_{i}\right)-F_{\Xi_{k}}\left(t-T_{0}-\sum_{i=1}^{k} d_{i}\right) .
\end{aligned}
$$

If $k>C_{t}$, then $t<T_{0}+\sum_{i=1}^{k} d_{i}$ and $P\left(W_{k}(t)\right)=0$.

Now if $k \leq C_{t}$, then $t-T_{0}-\sum_{i=1}^{k} d_{i} \geq 0$ and from (19) and Proposition 1 we get

$$
P\left(W_{k}(t)\right)=\frac{\gamma\left(\sum_{i=1}^{k} \alpha_{i}, \lambda\left(t-T_{0}-\sum_{i=1}^{k-1} d_{i}\right)\right)}{\Gamma\left(\sum_{i=1}^{k} \alpha_{i}\right)}-\frac{\gamma\left(\sum_{i=1}^{k} \alpha_{i}, \lambda\left(t-T_{0}-\sum_{i=1}^{k} d_{i}\right)\right)}{\Gamma\left(\sum_{i=1}^{k} \alpha_{i}\right)} .
$$


Remark 5. Note that in the case $d_{k}=0, k=1,2, \ldots$ from (20) it follows that $P\left(W_{k}(t)\right)=0$ for any $t \geq t_{0}$ and $k=1,2, \ldots$, i.e., we obtain the case of instantaneous random impulses.

Corollary 5. (Upper bound of $W_{k}(t)$ ). Let conditions (H3), (H4) hold and $t>T_{0}$ be a given fixed number. Then for $k: 1 \leq k \leq C_{t}$

$$
P\left(W_{k}(t)\right) \leq d_{k} e^{-\lambda\left(t-T_{0}-\sum_{i=1}^{k} d_{i}\right)} \frac{\left(\lambda\left(t-T_{0}\right)\right)^{\sum_{i=1}^{k} \alpha_{i}}}{\Gamma\left(\sum_{i=1}^{k} \alpha_{i}\right)\left(t-T_{0}-\sum_{i=1}^{k-1} d_{i}\right)},
$$

where $C_{t}=\min \left\{c \in \mathbb{Z}: t \leq T_{0}+\sum_{i=1}^{c} d_{i}\right\}$.

Proof: Applying the Integral Mean Value Theorem and the inequalities $t-T_{0}-\sum_{i=1}^{k-1} d_{i}<t-T_{0}$ we obtain

$$
\begin{aligned}
& \gamma\left(\sum_{i=1}^{k} \alpha_{i}, \lambda\left(t-T_{0}-\sum_{i=1}^{k-1} d_{i}\right)\right)-\gamma\left(\sum_{i=1}^{k} \alpha_{i}, \lambda\left(t-T_{0}-\sum_{i=1}^{k} d_{i}\right)\right) \\
& =\int_{0}^{\lambda\left(t-T_{0}-\sum_{i=1}^{k-1} d_{i}\right)} x^{\sum_{i=1}^{k} \alpha_{i}-1} e^{-x} d x-\int_{0}^{\lambda\left(t-T_{0}-\sum_{i=1}^{k} d_{i}\right)} x^{\sum_{i=1}^{k} \alpha_{i} m-1} e^{-x} d x \\
& =\int_{\lambda\left(t-T_{0}-\sum_{i=1}^{k-1} d_{i}\right)} x^{\sum_{i=1}^{k} \alpha_{i}-1} e^{-x} d x \\
& \leq \lambda\left(t-T_{0}-\sum_{i=1}^{k} d_{i}\right) \\
& \leq \lambda d_{k}\left(\lambda\left(t-T_{0}-\sum_{i=1}^{k-1} d_{i}\right)\right)^{\sum_{i=1}^{k} \alpha_{i}-1} e^{-\lambda\left(t-T_{0}-\sum_{i=1}^{k} d_{i}\right)} \\
& \leq d_{k} e^{-\lambda\left(t-T_{0}-\sum_{i=1}^{k} d_{i}\right)} \frac{\left(\lambda\left(t-T_{0}\right)\right)^{\sum_{i=1}^{k} \alpha_{i}}}{\left(t-T_{0}-\sum_{i=1}^{k-1} d_{i}\right)} .
\end{aligned}
$$

Corollary 6. Let conditions (H3), (H4) be satisfied with $\alpha_{i}=\alpha, i=1,2, \ldots$ and $t \geq T_{0}$ be a given fixed number. Then the probability that the time $t$ is immediately after the $k$-th random impulse but not further away than $d_{k}$ from it is given by

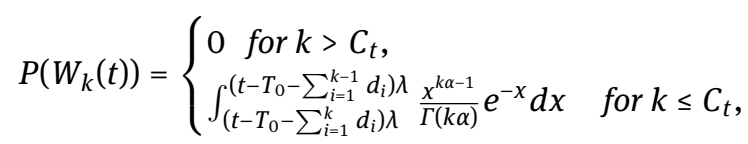

where $C_{t}=\min \left\{c \in \mathbb{Z}: t \leq T_{0}+\sum_{i=1}^{c} d_{i}\right\}$.

Corollary 7. If the condition (H3) is satisfied with $\alpha_{k}=1$, i.e., $\tau_{k} \in \operatorname{Exp}(\lambda)$ and $d_{k}=d>0, k=1,2, \ldots$, $t \geq T_{0}$ be a given fixed number, then

$$
P\left(W_{k}(t)\right)=\left\{\begin{array}{l}
0 \text { for } k>C_{t}, \\
\lambda d \frac{\left(\lambda\left(t-T_{0}-(k-1) d\right)\right)^{k-1} e^{-\lambda\left(t-T_{0}-k d\right)}}{k !} \text { for } k \leq C_{t},
\end{array}\right.
$$

where $C_{t}=\min \left\{c \in \mathbb{Z}: t \leq T_{0}+c d\right\}$.

Lemma 4.6. Let conditions (H3),(H5) be satisfied and $t \geq T_{0}$ be a given fixed number. Then the probability the time $t$ is immediately after the $k$-th random impulse but not further away than $d_{k}$ from it is given by 
- for $t \geq B$ :

$$
P\left(W_{k}(t)\right)=\frac{\gamma\left(\sum_{i=1}^{k} \alpha_{i}, \lambda\left(t-T_{0}-\sum_{i=1}^{k-1} d_{i}\right)\right)}{\Gamma\left(\sum_{i=1}^{k} \alpha_{i}\right)}-\frac{\gamma\left(\sum_{i=1}^{k} \alpha_{i}, \lambda\left(t-T_{0}-\sum_{i=1}^{k} d_{i}\right)\right)}{\Gamma\left(\sum_{i=1}^{k} \alpha_{i}\right)}
$$

- for $t<B$ :

$$
P\left(W_{k}(t)\right)= \begin{cases}0 \quad \text { for } k>C_{t}, & \\ \frac{\gamma\left(\sum_{i=1}^{k} \alpha_{i}, \lambda\left(t-T_{0}-\sum_{i=1}^{k-1} d_{i}\right)\right)}{\Gamma\left(\sum_{i=1}^{k} \alpha_{i}\right)}-\frac{\gamma\left(\sum_{i=1}^{k} \alpha_{i}, \lambda\left(t-T_{0}-\sum_{i=1}^{k} d_{i}\right)\right)}{\Gamma\left(\sum_{i=1}^{k} \alpha_{i}\right)} & \text { for } k \leq C_{t},\end{cases}
$$

where $C_{t}=\min \left\{c \in \mathbb{Z}: t \leq T_{0}+\sum_{i=1}^{c} d_{i}\right\}$.

The proof of Lemma 4.6 is similar to the one of Lemma 4.4, so we omit it.

Corollary 8. (Upper bound of $W_{k}(t)$ ). Let conditions (H3), (H5) hold and $t>T_{0}$ be a given fixed number. Then

$$
P\left(W_{k}(t)\right) \leq e^{\lambda B} \lambda d_{k} \frac{\left(\lambda\left(t-T_{0}-\sum_{i=1}^{k-1} d_{i}\right)\right)^{\sum_{i=1}^{k} \alpha_{i}-1}}{\Gamma\left(\sum_{i=1}^{k} \alpha_{i}\right)} e^{-\lambda\left(t-T_{0}\right)} .
$$

\section{Linear differential equations with random moments of non-instantaneous impulses}

We now obtain a formula for the solution of the initial value problem for a scalar linear differential equation with random non-instantaneous moments of impulses:

$$
\begin{aligned}
& u^{\prime}=-m_{k} u \quad \text { for } \quad \xi_{k}+d_{k}<t<\xi_{k+1}, k=0,1,2, \ldots, \\
& u(t)=b_{k} u\left(\xi_{k}\right) \text { for } \xi_{k}<t<\xi_{k}+d_{k}, k=1,2, \ldots, \\
& u\left(T_{0}\right)=u_{0},
\end{aligned}
$$

where $u_{0} \in \mathbb{R}, m_{k}>0,(k=0,1,2 \ldots)$ and $b_{k} \neq 1,(k=, 2, \ldots)$ are real constants.

Lemma 5.1. Let the following conditions be satisfied:

1. Condition (H3) is fulfilled.

2. The inequalities $m_{i}+\lambda \geq m_{k}$ hold for all natural $i, k$, such that $i<k$.

Then the solution of the IVP for the scalar linear differential equation with random non-instantaneous moments of impulses (21) is:

$$
u\left(t ; T_{0}, u_{0},\left\{\tau_{k}\right\}\right)=\left\{\begin{array}{l}
u_{0} e^{-\sum_{i=0}^{k-1} m_{i} \tau_{i+1}}\left(\prod_{i=1}^{k} b_{i}\right) \quad \text { for } \xi_{k}<t \leq \xi_{k}+d_{k}, k=1,2, \ldots, \\
u_{0} e^{-\sum_{i=0}^{k-1} m_{i} \tau_{i+1}}\left(\prod_{i=1}^{k} b_{i}\right) e^{-m_{k}\left(t-\xi_{k}-d_{k}\right)} \quad \text { for } \xi_{k}+d_{k}<t<\xi_{k+1}, k=0,1,2, \ldots,
\end{array}\right.
$$

and the expected value of the solution is:

$$
\begin{aligned}
E\left(\left|u\left(t ; T_{0}, u_{0},\left\{\tau_{k}\right\}\right)\right|\right)= & \left|u_{0}\right| e^{-m_{0}\left(t-T_{0}\right)} P\left(S_{0}(t)\right)+\sum_{k=1}^{\infty}\left|u_{0}\right|\left(\prod_{i=1}^{k}\left|b_{i}\right|\right)\left(\prod_{i=1}^{k}\left(\frac{\lambda}{m_{i-1}+\lambda}\right)^{\alpha_{i}}\right) P\left(W_{k}(t)\right) \\
& +\sum_{k=1}^{\infty}\left|u_{0}\right|\left(\prod_{i=1}^{k}\left|b_{i}\right|\right) e^{-m_{k}\left(t-T_{0}-\sum_{i=1}^{k} d_{i}\right)} \prod_{i=1}^{k}\left(\frac{\lambda}{m_{i-1}-m_{k}+\lambda}\right)^{\alpha_{i}} P\left(S_{k}(t)\right) .
\end{aligned}
$$


Proof: Let the point $t_{k}$ be an arbitrary value of $\tau_{k}, k=1,2, \ldots$ Define as above the points $T_{k}=T_{0}+\sum_{i=1}^{k} t_{i}+$ $\sum_{i=1}^{k-1} d_{i}$ which are values of the random variables $\xi_{k}$ defined by (8). Consider the linear scalar NIDE with fixed points of impulses:

$$
\begin{aligned}
& u^{\prime}=-m_{k} u \text { for } T_{k}+d_{k}<t<T_{k+1}, k=0,1,2, \ldots, \\
& u(t)=b_{k} u\left(T_{k}-0\right) \text { for } T_{k}<t<T_{k}+d_{k}, k=1,2, \ldots, \\
& u\left(T_{0}\right)=u_{0} .
\end{aligned}
$$

The solution of (23) is a sample path solution of (21). The solution of NIDE (23) is given by

$u\left(t ; T_{0}, u_{0},\left\{T_{k}\right\}\right)=\left\{\begin{array}{l}u_{0} e^{-\sum_{i=0}^{k-1} m_{i}\left(T_{i+1}-T_{i}-d_{i}\right)}\left(\prod_{i=1}^{k} b_{i}\right) \quad \text { for } T_{k}<t \leq T_{k}+d_{k}, k=1,2, \ldots, \\ u_{0} e^{-\sum_{i=0}^{k-1} m_{i}\left(T_{i+1}-T_{i}-d_{i}\right)}\left(\prod_{i=1}^{k} b_{i}\right) e^{-m_{k}\left(t-T_{k}-d_{k}\right)} \quad \text { for } T_{k}+d_{k}<t<T_{k+1}, k=0,1,2, \ldots\end{array}\right.$

Formula (24) and Definition 2 establishes (22).

From formula (22) and the independence of the random variables $\tau_{k}$ we obtain

$$
\begin{aligned}
E\left(\left|u\left(t ; T_{0}, u_{0},\left\{\tau_{k}\right\}\right)\right|\right)= & \left|u_{0}\right| e^{-m_{0}\left(t-T_{0}\right)} P\left(S_{0}(t)\right)+\sum_{k=1}^{\infty} E\left(\left|u\left(t ; T_{0}, u_{0},\left\{\tau_{k}\right\}\right)\right| \mid S_{k}(t)\right) P\left(S_{k}(t)\right) \\
& +\sum_{k=1}^{\infty} E\left(\left|u\left(t ; T_{0}, u_{0},\left\{\tau_{k}\right\}\right)\right| \mid W_{k}(t)\right) P\left(W_{k}(t)\right) \\
= & \left|u_{0}\right| e^{-m_{0}\left(t-T_{0}\right)}\left(\tau_{1}>t-T_{0}\right) P\left(S_{0}(t)\right)+\sum_{k=1}^{\infty}\left|u_{0}\right|\left(\prod_{i=1}^{k}\left|b_{i}\right|\right) E\left(e^{-\sum_{i=1}^{k} m_{i-1} \tau_{i}}\right) P\left(W_{k}(t)\right) \\
& +\sum_{k=1}^{\infty}\left|u_{0}\right|\left(\prod_{i=1}^{k}\left|b_{i}\right|\right) E\left(e^{-\sum_{i=0}^{k-1} m_{i} \tau_{i+1}} e^{-m_{k}\left(t-\xi_{k}-d_{k}\right)}\right) P\left(S_{k}(t)\right) \\
= & \left|u_{0}\right| e^{-m_{0}\left(t-T_{0}\right)} P\left(S_{0}(t)\right)+\sum_{k=1}^{\infty}\left|u_{0}\right|\left(\prod_{i=1}^{k}\left|b_{i}\right|\right) E\left(e^{-\sum_{i=0}^{k-1} m_{i} \tau_{i+1}}\right) P\left(W_{k}(t)\right) \\
& +\sum_{k=1}^{\infty}\left|u_{0}\right|\left(\prod_{i=1}^{k}\left|b_{i}\right|\right) e^{-m_{k}\left(t-T_{0}-\sum_{i=1}^{k} d_{i}\right)} E\left(e^{\sum_{i=1}^{k}\left(m_{k}-m_{i-1}\right) \tau_{i}}\right) P\left(S_{k}(t)\right) .
\end{aligned}
$$

Using the definition of the density function of the Gamma distribution and substituting $\left(m_{i-1}+\lambda\right) x=s$ we get

$$
\begin{aligned}
E e^{-m_{i-1} \tau_{i}} & =\int_{0}^{\infty} e^{-m_{i-1} x} \frac{\lambda^{\alpha_{i}} x^{\alpha_{i}-1} e^{-\lambda x}}{\Gamma\left(\alpha_{i}\right)} d x \\
& =\frac{\lambda^{\alpha_{i}}}{\Gamma\left(\alpha_{i}\right)} \int_{0}^{\infty} e^{-\left(m_{i-1}+\lambda\right) x} x^{\alpha_{i}-1} d x \\
& =\frac{1}{\left(m_{i-1}+\lambda\right)^{\alpha_{i}}} \frac{(\lambda)^{\alpha_{i}}}{\left(\alpha_{i}-1\right) !} \int_{0}^{\infty} e^{-s} s^{\alpha_{i}-1} d s \\
& =\left(\frac{\lambda}{m_{i-1}+\lambda}\right)^{\alpha_{i}}
\end{aligned}
$$


and substituting $\left(m_{i-1}-m_{k}+\lambda\right) x=s$ we get

$$
\begin{aligned}
E e^{\left(m_{k}-m_{i-1}\right) \tau_{i}} & =\int_{0}^{\infty} e^{\left(m_{k}-m_{i-1}\right) x} \frac{\lambda^{\alpha_{i}} x^{\alpha_{i}-1} e^{-\lambda x}}{\Gamma\left(\alpha_{i}\right)} d x \\
& =\frac{\lambda^{\alpha_{i}}}{\Gamma\left(\alpha_{i}\right)} \int_{0}^{\infty} e^{-\left(m_{i-1}-m_{k}+\lambda\right) x} x^{\alpha_{i}-1} d x \\
& =\frac{1}{\left(m_{i-1}-m_{k}+\lambda\right)^{\alpha_{i}}} \frac{(\lambda)^{\alpha_{i}}}{\left(\alpha_{i}-1\right) !} \int_{0}^{\infty} e^{-s} s^{\alpha_{i}-1} d s \\
& =\left(\frac{\lambda}{m_{i-1}-m_{k}+\lambda}\right)^{\alpha_{i}} .
\end{aligned}
$$

From (27) we obtain

$$
E\left(e^{\sum_{i=1}^{k}\left(m_{k}-m_{i-1}\right) \tau_{i}}\right)=\prod_{i=1}^{k}\left(\frac{\lambda}{m_{i-1}-m_{k}+\lambda}\right)^{\alpha_{i}} .
$$

Substitute (26) and (28) in (25) and obtain (23).

Corollary 9. Let the conditions of Lemma 5.1 be satisfied. Then for any $t \geq T_{0}$

$$
\begin{aligned}
E\left(\left|u\left(t ; T_{0}, u_{0},\left\{\tau_{k}\right\}\right)\right|\right)= & \left|u_{0}\right| e^{-m_{0}\left(t-T_{0}\right)}\left(1-\frac{\gamma\left(\alpha_{1},\left(t-T_{0}\right) \lambda\right)}{\Gamma\left(\alpha_{1}\right)}\right) \\
& +\sum_{k=1}^{C_{t}-1}\left|u_{0}\right| \prod_{i=1}^{k}\left(\left|b_{i}\right|\left(\frac{\lambda}{m_{i-1}+\lambda}\right)^{\alpha_{i}}\right)\left(\frac{\gamma\left(\sum_{i=1}^{k} \alpha_{i}, \lambda\left(t-T_{0}-\sum_{i=1}^{k-1} d_{i}\right)\right)}{\Gamma\left(\sum_{i=1}^{k} \alpha_{i}\right)}\right. \\
& \left.-\frac{\gamma\left(\sum_{i=1}^{k} \alpha_{i}, \lambda\left(t-T_{0}-\sum_{i=1}^{k} d_{i}\right)\right)}{\Gamma\left(\sum_{i=1}^{k} \alpha_{i}\right)}\right) \\
& +\sum_{k=1}^{C_{t}-1}\left|u_{0}\right| e^{-m_{k}\left(t-T_{0}-\sum_{i=1}^{k} d_{i}\right)} \prod_{i=1}^{k}\left(\left|b_{i}\right|\left(\frac{\lambda}{m_{i-1}-m_{k}+\lambda}\right)^{\alpha_{i}}\right)\left(\frac{\gamma\left(\sum_{i=1}^{k} \alpha_{i},\left(t-T_{0}-\sum_{i=1}^{k} d_{i}\right) \lambda\right)}{\Gamma\left(\sum_{i=1}^{k} \alpha_{i}\right)}\right. \\
& \left.-\frac{\gamma\left(\sum_{i=1}^{k+1} \alpha_{i},\left(t-T_{0}-\sum_{i=1}^{k} d_{i}\right) \lambda\right)}{\Gamma\left(\sum_{i=1}^{k+1} \alpha_{i}\right)}\right),
\end{aligned}
$$

where $C_{t}=\min \left\{c \in \mathbb{Z}: t \leq T_{0}+\sum_{i=1}^{c} d_{i}\right\}$.

The proof follows from Proposition 3, Lemma 4.2, Lemma 4.5 and Lemma 5.1.

Corollary 10. Let the conditions of Lemma 5.1 be satisfied with $m_{k}=m, k=1,2, \ldots$. Then for any $t \geq T_{0}$

$$
\begin{aligned}
E\left(\left|u\left(t ; T_{0}, u_{0},\left\{\tau_{k}\right\}\right)\right|\right)= & \left|u_{0}\right| e^{-m\left(t-T_{0}\right)} P\left(S_{0}(t)\right)+\left|u_{0}\right| \sum_{k=1}^{\infty}\left(\prod_{i=1}^{k}\left|b_{i}\right|\left(\frac{\lambda}{m+\lambda}\right)^{\alpha_{i}}\right) P\left(W_{k}(t)\right) \\
& +\left|u_{0}\right| e^{-m\left(t-T_{0}\right)} \sum_{k=1}^{\infty}\left(\prod_{i=1}^{k}\left|b_{i}\right| e^{m d_{i}}\right) P\left(S_{k}(t)\right) .
\end{aligned}
$$

Lemma 5.2. (upper bound of the expected value). Let the conditions of Lemma 5.1 be satisfied. Then

$$
\begin{aligned}
E\left(\left|u\left(t ; T_{0}, u_{0},\left\{\tau_{k}\right\}\right)\right|\right)= & \left|u_{0}\right| e^{-m_{0}\left(t-T_{0}\right)} P\left(S_{0}(t)\right)+\left|u_{0}\right| e^{-\lambda\left(t-T_{0}\right)} \sum_{k=1}^{C_{t}-1} d_{k} \frac{\left(\prod_{i=1}^{k}\left|b_{i}\right| e^{\lambda d_{i}}\left(\frac{\lambda^{2}\left(t-T_{0}\right)}{m_{i-1}+\lambda}\right)^{\alpha_{i}}\right.}{\Gamma\left(\sum_{i=1}^{k} \alpha_{i}\right)\left(t-T_{0}-\sum_{i=1}^{k-1} d_{i}\right)} \\
& +\left|u_{0}\right| \sum_{k=1}^{\infty} e^{-m_{k}\left(t-T_{0}\right)}\left(\prod_{i=1}^{k}\left|b_{i}\right| e^{m_{k} d_{i}}\left(\frac{\lambda}{m_{i-1}-m_{k}+\lambda}\right)^{\alpha_{i}}\right) P\left(S_{k}(t)\right),
\end{aligned}
$$


where $C_{t}=\min \left\{c \in \mathbb{Z}: t \leq T_{0}+\sum_{i=1}^{c} d_{i}\right\}$.

The proof follows from Lemma 5.1 and Corollary 5.

Corollary 11. (upper bound of the expected value) Let the conditions of Lemma 5.1 be satisfied.

If there exists a constant $M \geq 1$, such that

$$
\begin{aligned}
& \sum_{k=1}^{C_{t}-1} d_{k} \frac{\prod_{i=1}^{k}\left(e^{\lambda d_{i}}\left|b_{i}\right|\left(\frac{\lambda^{2}\left(t-T_{0}\right)}{m_{i-1}+\lambda}\right)^{\alpha_{i}}\right)}{\left(t-T_{0}-\sum_{i=1}^{k-1} d_{i}\right) \Gamma\left(\sum_{i=1}^{k} \alpha_{i}\right)} \leq M, \\
& \quad t>T_{0}
\end{aligned}
$$

and

$$
\begin{aligned}
& \prod_{i=1}^{k}\left(e^{m_{k} d_{i}}\left|b_{i}\right|\left(\frac{\lambda}{m_{i-1}-m_{k}+\lambda}\right)^{\alpha_{i}}\right) \leq M, \\
& \quad k=1,2, \ldots
\end{aligned}
$$

then

$$
E\left(\left|u\left(t ; T_{0}, u_{0},\left\{\tau_{k}\right\}\right)\right|\right) \leq\left|u_{0}\right| e^{-m_{0}\left(t-T_{0}\right)} P\left(S_{0}(t)\right)+\left|u_{0}\right| e^{-\lambda\left(t-T_{0}\right)} M+\left|u_{0}\right| \sum_{k=1}^{C_{t}-1} e^{-m_{k}\left(t-T_{0}\right)} M P\left(S_{k}(t)\right) .
$$

If additionally there exists a constant $m^{\star}>0$, such that $\lambda \geq m^{\star}, m_{i} \geq m^{\star}, i=1,2, \ldots, k$, then

$$
E\left(\left|u\left(t ; T_{0}, u_{0},\left\{\tau_{k}\right\}\right)\right|\right) \leq\left|u_{0}\right| M e^{-m^{*}\left(t-T_{0}\right)}\left(1+\sum_{k=1}^{C_{t}-1} P\left(S_{k}(t)\right)\right) \leq 2\left|u_{0}\right| M e^{-m^{*}\left(t-T_{0}\right)} .
$$

\section{6 p-moment exponential stability for RNIDE}

The main goal of the paper is to define the exponential stability of the zero solution of RNIDE (10) (with $x_{0}=0$ ) and to obtain some sufficient conditions for it.

Definition 3. Let $p>0$. Then the trivial solution $\left(x_{0}=0\right)$ of the RNIDE $(10)$ is said to be $p$-moment exponentially stable if for any initial point $x_{0} \in \mathbb{R}^{n}$ there exist constants $\alpha, \mu>0$, such that $\left.E\left[\| x\left(t ; T_{0}, x_{0},\left\{\tau_{k}\right)\right\}\right) \|^{p}\right]<$ $\alpha\left\|x_{0}\right\|^{p} e^{-\mu\left(t-T_{0}\right)}$ for all $t>T_{0}$, where $x\left(t ; T_{0}, x_{0},\left\{\tau_{k}\right)\right\}$ is the solution of the IVP for the RNIDE (10).

Remark 6. We note that the two-moment exponential stability for stochastic equations is known as exponential stability in mean square.

In this section we will use Lyapunov functions to obtain sufficient conditions for the p-moment exponential stability of the trivial solution of nonlinear system with impulses occurring at random moments and acting non-instantaneously.

Definition 4. Let $J \subset \mathbb{R}_{+}$be a given interval and $\Delta \subset \mathbb{R}^{n}, 0 \in \Delta$ be a given set. We will say that the function $V(t, x): J \times \Delta \rightarrow \mathbb{R}_{+}, V(t, 0) \equiv 0$ belongs to the class $\Lambda(J, \Delta)$ if it is continuous on $J \times \Delta$ and locally Lipschitzian with respect to its second argument.

For functions $V(t, x) \in \Lambda(J, \Delta)$ we will use Dini derivatives defined by :

$$
D_{+} V(t, x)=\limsup _{h \rightarrow 0^{+}} \frac{1}{h}\{V(t, x)-V(t-h, x-h f(t, x))\} \text { for } t \in J, x \in \Delta,
$$

where there exists $h_{1}>0$, such that $t-h \in J, x-h f(t, x) \in \Delta$ for $0<h \leq h_{1}$. 
Theorem 6.1. Let the following conditions be satisfied:

1. Conditions (H1), (H2), (H3), (H4) hold.

2. The function $V \in \Lambda\left(\left[T_{0}, \infty\right), \mathbb{R}^{n}\right)$ and there exist positive constants $a, b$, such that

(i) $a\|x\|^{p} \leq V(t, x) \leq b\|x\|^{p}$ for $t \geq T_{0}, x \in \mathbb{R}^{n}$;

(ii) there exists a constant $m$ satisfying $0<m \leq \lambda$, such that the inequality

$$
D_{+} V(t, x) \leq-m V(t, x), \text { for } t>T_{0}, x \in \mathbb{R}^{n}
$$

holds;

(iii) for any $k=1,2, \ldots$ there exist functions $w_{k} \in C\left(\mathbb{R}_{+}, \mathbb{R}_{+}\right)$, such that

$$
V\left(t, I_{k}(x)\right) \leq w_{k}(t) V(t, x) \quad \text { for } t \geq T_{0}, x \in \mathbb{R}^{n} .
$$

3. There exist positive constants $M \geq 1$ and $C_{k}<1, k=1,2, \ldots$, such that $w_{k}(t) \leq C_{k}$ for $t \geq T_{0}$ and for $t>T_{0}$ the following hold

$$
2 \lambda \sum_{k=1}^{\infty} d_{k} e^{\lambda \sum_{i=1}^{k} d_{i}}\left(\lambda\left(t-T_{0}\right)\right)^{\sum_{i=1}^{k} \alpha_{i}-1} \prod_{i=1}^{k} C_{i} \leq M,
$$

and

$$
\prod_{i=1}^{k} e^{m d_{i}} C_{i} \leq M, k=1,2, \ldots
$$

Then the trivial solution of the RNIDE (10) is p-moment exponentially stable.

Proof: Let $x_{0} \in \mathbb{R}^{n}$ be an arbitrary initial point and the stochastic process $x_{\tau}(t)=x\left(t ; T_{0}, x_{0},\left\{\tau_{k}\right)\right\}$ be a solution of the initial value problem for the RNIDE (10).

Now consider the IVP for the scalar linear RNIDE (21) with $m_{k}=m, b_{k}=C_{k}$ for $k=1,2, \ldots$, and $x_{0}=V\left(T_{0}, x_{0}\right)$. According to Lemma 5.1 the solution $u\left(t ; T_{0}, V\left(T_{0}, x_{0}\right),\left\{\tau_{k}\right\}\right)$ of RNIDE (21) is given by (22).

Let $t_{k}$ be an arbitrary value of the random variable $\tau_{k}, k=1,2, \ldots$ Then $T_{k}=T_{0}+\sum_{i=1}^{k} t_{i}+\sum_{i=1}^{k} d_{i}, k=$ $1,2, \ldots$ are values of the random variables $\xi_{k}$. Thus the corresponding function $x(t)=x\left(t ; T_{0}, x_{0},\left\{T_{k}\right\}\right)$ is a sample path solution of the IVP for RNIDE (10), i.e., $x(t)=x\left(t ; T_{0}, x_{0},\left\{T_{k}\right\}\right)$ is a solution of the IVP for the NIDE with fixed points of impulses (1).

For any given $\left\{T_{k}\right\}$ and $x_{0}$ we define

$$
v(t)=V\left(t, x\left(t ; T_{0}, x_{0},\left\{T_{k}\right\}\right)\right), t \geq T_{0}, t \neq T_{k}
$$

and

$$
v\left(T_{k}\right)=V\left(T_{k}, x\left(T_{k}-0 ; T_{0}, x_{0},\left\{T_{k}\right\}\right), k=1,2, \ldots\right.
$$

Let $t \in\left(T_{k}, s_{k}\right], k=0,1,2, \ldots$ Using the continuity and monotonicity of the function $V(t, x)$ and condition 2(iii) we obtain

$$
\begin{aligned}
v(t) & =V(t, x(t))=V\left(t, I_{k}\left(x\left(T_{k}-0\right)\right)\right) \\
& \leq w_{k}(t) V\left(t, x\left(T_{k}-0\right)\right) \leq w_{k}(t) V\left(T_{k}, x\left(T_{k}-0\right)\right) \\
& =w_{k}(t) v\left(T_{k}-0\right) \\
& \leq C_{k} v\left(T_{k}-0\right), \quad t \in\left(T_{k}, s_{k}\right], k=0,1,2, \ldots
\end{aligned}
$$

Now, consider any interval $\left(s_{k}, T_{k+1}\right]$. Using $v\left(s_{k}\right)=V\left(s_{k}, x\left(s_{k} ; T_{0}, x_{0},\left\{T_{k}\right\}\right)\right)$ we obtain

$$
\begin{aligned}
v^{\prime}(t)=D_{+} v(t) & =D_{+} V\left(t, x\left(t ; T_{0}, x_{0},\left\{T_{k}\right\}\right)\right) \\
& \leq-m V\left(t, x\left(t ; T_{0}, x_{0},\left\{T_{k}\right\}\right)\right)=-m v(t), \quad t \in\left(s_{k}, T_{k+1}\right] .
\end{aligned}
$$


Therefore, from (37) and (38) it follows that the function $v(t)$ satisfies the linear impulsive differential inequalities with fixed points of non-instantaneous impulses:

$$
\begin{aligned}
& v^{\prime}(t) \leq-m v(t) \text { for } s_{k}<t<T_{k+1}, k=1,2, \ldots, \\
& v\left(T_{k}+\right) \leq C_{k} v\left(T_{k}\right) \text { for } T_{k}<t \leq s_{k}, k=1,2, \ldots, \\
& v\left(T_{0}\right)=V\left(T_{0}, x_{0}\right) .
\end{aligned}
$$

Consider the function

$$
m(t)=v(t)-u\left(t ; T_{0}, V\left(T_{0}, x_{0}\right),\left\{\tau_{k}\right\}\right) \text { for } t \geq T_{0} .
$$

The function $m(t)$ is piecewise continuous and according to Proposition 1 it is non-positive on the interval $\left[T_{0}, \infty\right)$, i.e.,

$$
v(t) \leq u\left(t ; T_{0}, x_{0},\left\{T_{k}\right\}\right) \text { for } t \geq T_{0} .
$$

Note that inequality (40) is satisfied for any arbitrary given sequence of fixed points of impulses $\left\{T_{k}\right\}$. Therefore, the generated by $v(t)$ stochastic process $v_{\tau}(t)$ satisfies the inequality $v_{\tau}(t) \leq u\left(t ; T_{0}, x_{0},\left\{\tau_{k}\right\}\right)$.

Inequality (36) proves the validity of (31) with $m_{i}=m$ and $b_{i}=C_{i}$. From condition 3 we obtain

$$
\begin{aligned}
\sum_{k=1}^{C_{t}-1} d_{k} \frac{\prod_{i=1}^{k}\left(e^{\lambda d_{i}} C_{i}\left(\frac{\lambda^{2}\left(t-T_{0}\right)}{m+\lambda}\right)^{\alpha_{i}}\right)}{\left(t-T_{0}-\sum_{i=1}^{k-1} d_{i}\right) \Gamma\left(\sum_{i=1}^{k} \alpha_{i}\right)} & \leq 2 \sum_{k=1}^{C_{t}-1} d_{k} \frac{\prod_{i=1}^{k}\left(e^{\lambda d_{i}} C_{i}\left(\lambda\left(t-T_{0}\right)\right)^{\alpha_{i}}\right)}{\left(t-T_{0}-\sum_{i=1}^{k-1} d_{i}\right)} \\
& \leq 2 \sum_{k=1}^{\infty} d_{k} \frac{\prod_{i=1}^{k}\left(e^{\lambda d_{i}} C_{i}\left(\lambda\left(t-T_{0}\right)\right)^{\alpha_{i}}\right)}{\left(t-T_{0}-\sum_{i=1}^{k-1} d_{i}\right)} \leq M, \quad t>T_{0} .
\end{aligned}
$$

Therefore, inequality (30) holds with $m_{i}=m$ and $b_{i}=C_{i}$.

From Corollary 11 and inequality (33) with $m^{\star}=m$ and condition 2(i) of Theorem 6.1 we obtain the inequalities

$$
\begin{aligned}
E\left(\left\|x_{\tau}(t)\right\|^{p}\right) & =\frac{1}{a} E\left(a\left\|x_{\tau}(t)\right\|^{p}\right) \leq \frac{1}{a} E\left(V\left(t, x_{\tau}(t)\right)\right) \leq \frac{1}{a} E\left(v_{\tau}(t)\right) \\
& \leq \frac{1}{a} E\left(u\left(t ; T_{0}, x_{0},\left\{\tau_{k}\right\}\right)\right) \leq \frac{2 M}{a} V\left(T_{0}, x_{0}\right) e^{-m\left(t-T_{0}\right)} \leq \frac{2 M b}{a}\left\|x_{0}\right\|^{p} e^{-m\left(t-T_{0}\right)}, \quad t \geq T_{0} .
\end{aligned}
$$

Inequality (42) proves the p-moment exponential stability.

Remark 7. Note that the inequalities (35) and (36) are satisfied, for example, for $C_{i}=e^{-\lambda d_{i}}, d_{i}=\frac{1}{i}, \alpha_{i}=e^{-i}$, because $\sum_{i=1}^{k} \alpha_{i}=\frac{1-e^{-k}}{e-1}<1$ for $k \geq 1$.

Theorem 6.2. Let :

1. Conditions (H1), (H2), (H3), (H5) hold.

2. Condition 2 of Theorem 6.1 be satisfied.

3. There exists a positive constant $C<1$, such that $w_{k}(t) \leq C$ for $t \geq T_{0}$ and

$$
2 B \lambda \sum_{k=1}^{\infty}\left(\lambda\left(t-T_{0}\right)\right)^{\sum_{i=1}^{k} \alpha_{i}-1} \leq e^{m B} \quad \text { for any } t>T_{0} .
$$

Then the trivial solution of the RNIDE (10) is p-moment exponentially stable.

Proof: Choose $M=e^{m \sum_{i=1}^{\infty} d_{i}}=e^{m B}>1$. Then $C e^{m \sum_{i=1}^{k} d_{i}} \leq C e^{m \sum_{i=1}^{\infty} d_{i}}=C e^{m B} \leq M$, i.e., inequality (31) holds with $m_{i}=m, b_{i}=C$. 
Using $\Gamma(x) \geq 0.5$ for $x \geq 0$ and $\frac{\lambda}{m+\lambda}<1$ we have

$$
\begin{aligned}
\sum_{k=1}^{C_{t}-1} d_{k} C^{k} \frac{\prod_{i=1}^{k}\left(e^{\lambda d_{i}}\left(\frac{\lambda^{2}\left(t-T_{0}\right)}{m+\lambda}\right)^{\alpha_{i}}\right)}{\left(t-T_{0}-\sum_{i=1}^{k-1} d_{i}\right) \Gamma\left(\sum_{i=1}^{k} \alpha_{i}\right)} & \leq 2 \sum_{k=1}^{C_{t}-1} C^{k} d_{k} \frac{\left(\lambda\left(t-T_{0}\right)\right)^{\sum_{i=1}^{k} \alpha_{i}}}{\left(t-T_{0}-\sum_{i=1}^{k-1} d_{i}\right)} \\
& \leq 2 \lambda \sum_{k=1}^{\infty} d_{k}\left(\lambda\left(t-T_{0}\right)\right)^{\sum_{i=1}^{k} \alpha_{i}-1} \\
& \leq 2 B \lambda \sum_{k=1}^{\infty}\left(\lambda\left(t-T_{0}\right)\right)^{\sum_{i=1}^{k} \alpha_{i}-1} \leq e^{m B}, \quad t>T_{0} .
\end{aligned}
$$

Therefore, inequality (30) holds with $m_{i}=m$ and $b_{i}=C$ and we can apply Corollary 11 . The rest of the proof is the same as the proof of Theorem 6.1 so we omit it.

Remark 8. Note that the inequality (43) is satisfied for example, for $\alpha_{i}=e^{-i}$, because $\sum_{i=1}^{k} \alpha_{i}=\frac{1-e^{-k}}{e-1}<1$ for $k \geq 1$.

Example 2. (p-exponential stability of RNIDE). Let $\tau_{i}, i=1,2, \ldots$ be independent Gamma distributed random variables with the same rate parameter $\lambda$ and shape parameter $\alpha_{k}$ respectively, i.e., $\tau_{k} \in \Gamma\left(\alpha_{k}, \lambda\right)$ with $\lambda \in(0,0.1], \alpha_{k}=e^{-k}$. Let $d_{k}=\frac{\pi}{4}$. Let the points $s_{k}=(4 k+1) \frac{\pi}{2}, t_{k}=(4 k-1) \frac{\pi}{2}, k=1,2, \ldots, s_{0}=0$. Consider the following initial value problem for the scalar non-instantaneous impulsive differential equation

$$
\begin{aligned}
& x^{\prime}(t)=x f(t) \text { for } t \geq 0, \xi_{k}+\frac{\pi}{2}<t<\xi_{k+1}, k=0,1,2, \ldots, \\
& x(t)=I_{k}(t) x\left(t_{k}-0\right) \quad \text { for } \xi_{k}<t<\xi_{k}+\frac{\pi}{2}, k=1,2, \ldots, \\
& x(0)=x_{0},
\end{aligned}
$$

where $x \in \mathbb{R}, I_{k}(t): \mathbb{R}_{+}^{n} \rightarrow \mathbb{R}^{n},\left|I_{k}(t)\right| \leq C_{k}, C_{k} \leq e^{-\frac{\pi}{40}}, f(t)=0.5 \frac{\cos (t)-\frac{t+1}{t+2}}{2-\sin (t)}, k=0,1,2, \ldots$

The zero solution of the corresponding $\operatorname{ODE} x^{\prime}(t)=x f(t)$ is exponentially stable (see Figure 1).

Also, to illustrate the behavior of the solution of (44) with $x_{0}=0.3, I_{k}(t)=\cos (t) e^{-\frac{\pi}{40}}$, we graph some of the path solutions for several randomly selected different values of $\tau_{i}, i=1,2, \ldots$, i.e. different values of the waiting time until the next impulse. Firstly, one value of each random variable $\tau_{i}, i=1,2,3,4$ is chosen, i.e., $\tau_{1}=0.5, \tau_{2}=1, \tau_{3}=5, \tau_{4}=0.4$ and the corresponding path solution is graphed on Figure 3 . Also, three values of each of the random variables $\tau_{i}, i=1,2,3,4$ are chosen and the corresponding path solutions are graphed on Figure 4, i.e., for values $\tau_{1}=0.5, \tau_{2}=1, \tau_{3}=5, \tau_{4}=0.4$ the graph of the path solution is the black dashed curve; for $\tau_{1}=1, \tau_{2}=0.5, \tau_{3}=3, \tau_{4}=2$ the graph of the path solution is the black curve, and for $\tau_{1}=5, \tau_{2}=1, \tau_{3}=6, \tau_{4}=0.5$ the graph of the path solution is the black thick curve. From these graphs it is seen the path solutions approaches 0 .

Let $V(t, x)=x^{2}$. Then $D_{+} V(t, x)=2 x^{2} f(t)$. Since the sign of the function $f(t)$ changes (see Figure 2) the quadratic Lyapunov function is not applicable to equation (44).

Let $V(t, x)=(2-\sin (t)) x^{2}$. The condition 2(i) of Theorem 6.1 is satisfied with $a=1, b=3$. Apply the Dini derivative and we get

$$
D_{+} V(t, x)=2 x^{2}(2-\sin (t)) f(t)-x^{2} \cos (t)=-\frac{0.5(t+1)}{(t+2)(2-\sin (t))} V(t, x) \leq-0.1 V(t, x),
$$

i.e., Condition 2 (ii) of Theorem 6.1 is satisfied with $m=0.1$.

Let $k=1,2, \ldots, x \in \mathbb{R}$ and we get $V\left(t, I_{k}(x)\right) \leq(2-\sin (t))\left(c_{k} x\right)^{2}=(2-\sin (t)) c_{k}^{2} x^{2}=c_{k}^{2} V(t, x)$, i.e., condition 2(ii) is satisfied. 


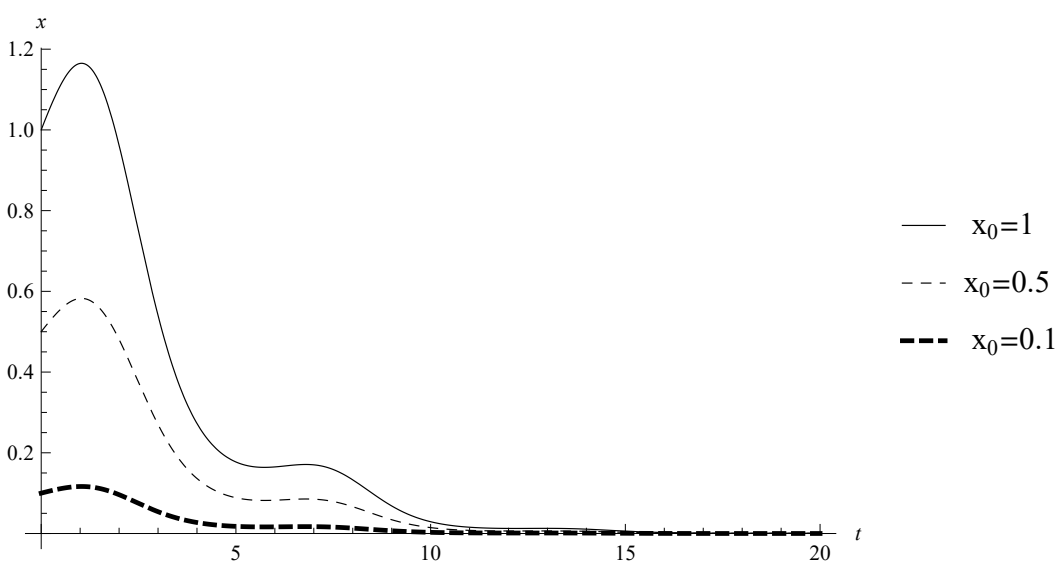

Figure 1: Solutions of $\operatorname{ODE} x^{\prime}(t)=0.5 x \frac{\cos (t)-\frac{t+1}{t+2}}{2-\sin (t)}$ with $x_{0}=0.1,0.5,0.1$

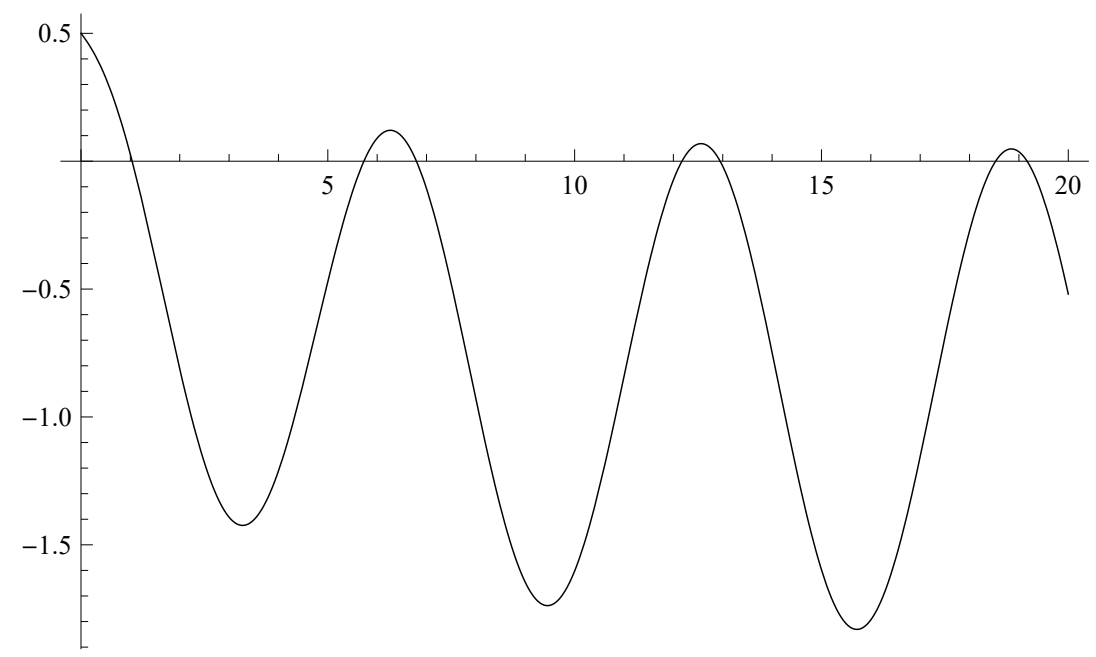

Figure 2: Graph of $f(t)=0.5 \frac{\cos (t)-\frac{t+1}{t+2}}{2-\sin (t)}$.

The inequalities (35) and (36) are satisfied with $d_{i}=\frac{\pi}{4}, m=0.1$ and $T_{0}=0$. Indeed,

$$
\begin{aligned}
2 \lambda \sum_{k=1}^{\infty} d_{k} e^{\lambda \sum_{i=1}^{k} d_{i}}\left(\lambda\left(t-T_{0}\right)\right)^{\sum_{i=1}^{k} \alpha_{i}-1} \prod_{i=1}^{k} C_{i} & \leq \lambda \frac{\pi}{2} \sum_{k=1}^{\infty} e^{(\lambda-0.1) \frac{k \pi}{4}}(\lambda t)^{\sum_{i=1}^{k} e^{-i}-1} \\
& =\lambda \frac{\pi}{2} \sum_{k=1}^{\infty} e^{(\lambda-0.1) \frac{k \pi}{4}}(\lambda t)^{\frac{1-e^{-k}}{e-1}-1}
\end{aligned}
$$

Since $\lambda-0.1 \leq 0$, and $\frac{1-e^{-k}}{e-1}<1$ for any $t>0$ there exists $M=M(t) \geq 1$, such that

$$
\frac{\lambda \pi}{2} \sum_{k=1}^{\infty} e^{(\lambda-0.1) \frac{k \pi}{4}}(\lambda t)^{\frac{1-e^{-k}}{e-1}-1} \leq M, \quad t>0
$$

and

$$
\prod_{i=1}^{k} e^{m d_{i}} C_{i} \leq \prod_{i=1}^{k} e^{\frac{\pi}{40}} C_{i} \leq 1 \leq M, k=1,2, \ldots
$$

From Theorem 6.1 the zero solution of (44) is exponentially stable in mean square, i.e., the random noninstantaneous impulses could preserve the stability properties of the zero solution. 


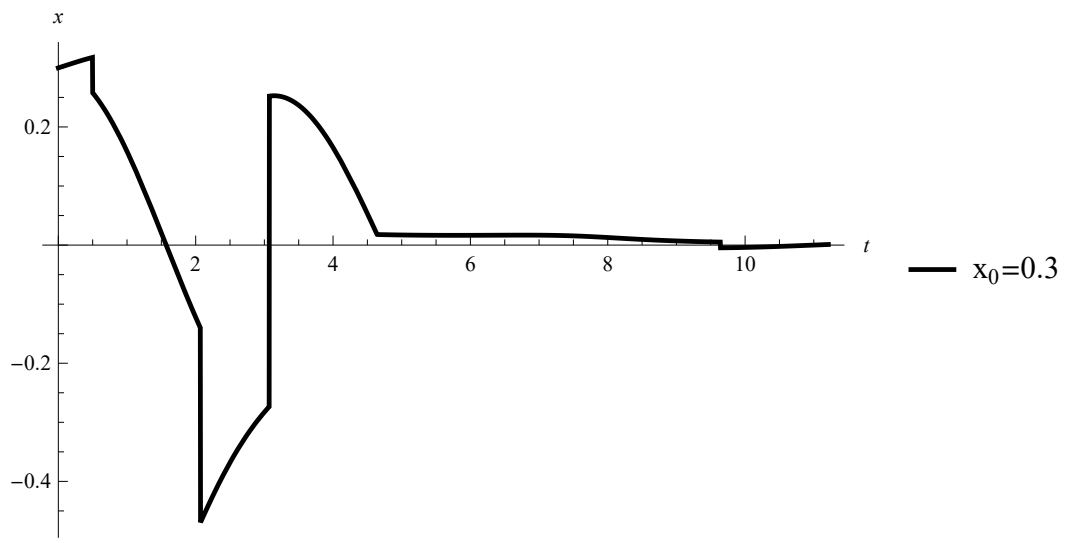

Figure 3: Graph of solution of (44) for one value of each random variable $\tau_{k}$.

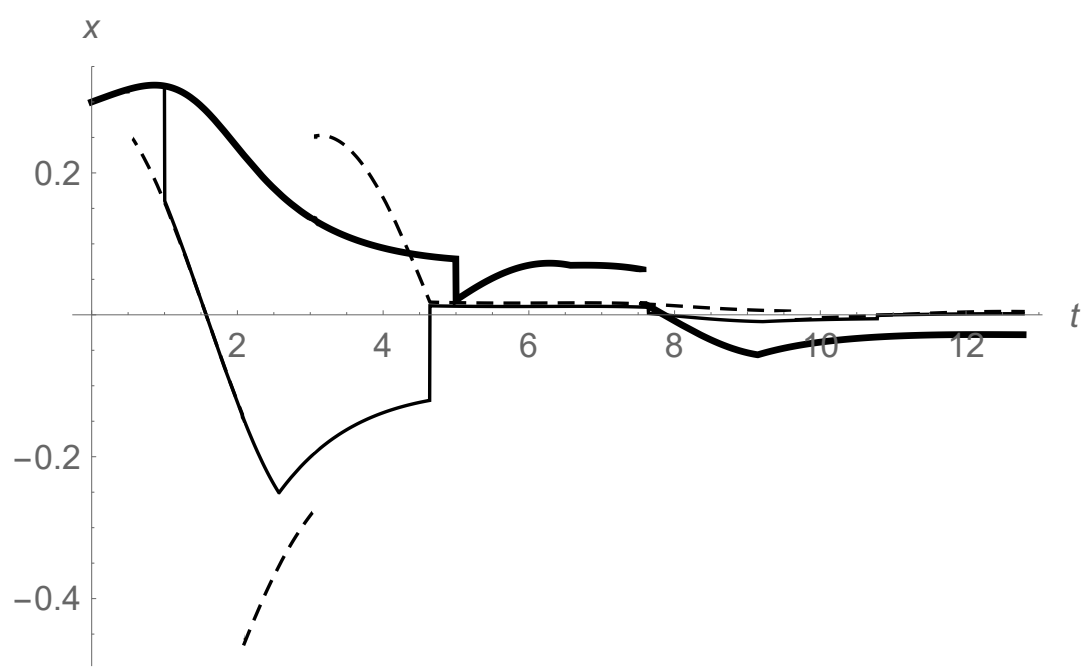

Figure 4: Graphs of solutions of (44) for various values of random variables $\tau_{k}$.

Acknowledgement: Research was partially supported by Fund PP18-FMI-003, Unversity of Plovdiv "Paisii Hilendarski".

\section{References}

[1] Hristova S., Integral stability in terms of two measures for impulsive functional differential equations, Math. Comput. Model., 2010, 51(1-2), 100-108

[2] Hristova S., Stability on a cone in terms of two measures for impulsive differential equations with "supremum", Appl. Math. Lett., 2010, 23(5), 508-511

[3] Hristova S., Razumikhin method and cone valued Lyapunov functions for impulsive differential equations with "supremum“, Int. J. Dyn. Syst. Differ. Equ., 2009, 2, 223-236

[4] Hristova S., Stefanova K., Practical stability of impulsive differential equations with "supremum" by integral inequalities, Eur. J. Pure Appl. Math., 2012, 5, 30-44

[5] Hristova S., Lipschitz stability for impulsive differential equations with “supremum“, Int. Electron. J. Pure Appl. Math., 2010, 1, 345-358

[6] Hristova S., Georgieva A., Practical stability in terms of two measures for impulsive differential equations with "supremum", Int. J. Differ. Equ., 2011, Article ID 703189

[7] Hristova S., Qualitative Investigations and Approximate Methods for Impulsive Differential Equations, Nova Science Publishers, New York, 2009 
[8] Lakshmikantham V., Bainov D. D., Simeonov P. S., Theory of Impulsive Differential Equations, World Scientific, Singapore, 1989

[9] Hernandez E., Pierri M., O’Regan D., On abstract differential equations with non instantaneous impulses, Topol. Methods Nonlinear Anal., 2015, 46(2), 1067-1088

[10] Agarwal R., O’Regan D., Hristova S., Stability by Lyapunov like functions of nonlinear differential equations with noninstantaneous impulses, J. Appl. Math. Comput., 2017, 53(1-2), 147-168

[11] Church K. E. M., Smith R. J., Existence and uniqueness of solutions of general impulsive extension equations with specification to linear equations, Dyn. Contin. Discrete Impuls. Syst. Ser. B Appl. Algorithms, 2015, 22, 163-197

[12] Liao Y. M., Wang J. R., A note on stability of impulsive differential equations, Bound. Value Probl., 2014, 67

[13] Das S., Pandey D. N., Sukavanam N., Existence of solution of impulsive second order neutral integro-differential equations with state delay, J. Integral Equations Appl., 2015, 27(4), 489-520

[14] Agarwal R., O’Regan D., Hristova S., Stability of Caputo fractional differential equations with non-instantaneous impulses, Commun. Appl. Anal., (accepted)

[15] Kumar P., Pandey D. N., Bahuguna D., On a new class of abstract impulsive functional differential equations of fractional order, J. Nonlinear Sci. Appl., 2014, 7(2), 102-114

[16] Li P., Xu Ch., Boundary value problems of fractional order differential equation with integral boundary conditions and not instantaneous impulses, J. Funct. Spaces, 2015, Article ID 954925

[17] Sanz-Serna J. M., Stuart A. M., Ergodicity of dissipative differential equations subject to random impulses, J. Differential Equations, 1999, 155, 262-284

[18] Wu S., Hang D., Meng X., p-moment stability of stochastic equations with jumps, Appl. Math. Comput., 2004, 152, 505-519

[19] Wu H., Sun J., p-moment stability of stochastic differential equations with impulsive jump and Markovian switching, Automatica, 2006, 42(10), 1753-1759

[20] Yang J., Zhong S., Luo W., Mean square stability analysis of impulsive stochastic differential equations with delays, J. Comput. Appl. Math., 2008, 216(2), 474-483

[21] Anguraj A., Vinodkumar A., Existence, uniqueness and stability results of random impulsive semilinear differential systems, Nonlinear Anal. Hybrid Syst., 2010, 4(3), 475-483

[22] Wang J. R., Feckan M., Zhou Y., Random noninstantaneous impulsive models for studying periodic evolution processes in pharmacotherapy, In: Luo A., Merdan H. (Eds.) Mathematical Modeling and Applications in Nonlinear Dynamics, Nonlinear Systems and Complexity, 2016, 14, 87-107

[23] Kolmogorov A. N., Fomin S. V., Introductory Real Analysis, Dover Publ. Inc., New York, 1970 